\title{
Backward uniqueness for general parabolic operators in the whole space
}

\author{
Jie $\mathrm{Wu}^{*}$ and Liqun Zhang $^{\dagger}$
}

October, 2017

\begin{abstract}
We prove the backward uniqueness for general parabolic operators of second order in the whole space under assumptions that the leading coefficients of the operator are Lipschitz and their gradients satisfy certain decay conditions. This result extends in some ways a classical result of Lions and Malgrange [12] and a recent result of the authors [10].
\end{abstract}

Keywords: Carleman estimates; Unique continuation; Backward uniqueness; Parabolic operator.

MSC (2010): 35K10; 35A02; 35R45.

\section{Introduction}

Let $P$ be a backward parabolic operator on $\mathbb{R}^{n} \times[0,1]$,

$$
P=\partial_{t}+\partial_{i}\left(a^{i j}(x, t) \partial_{j}\right)=\partial_{t}+\nabla \cdot(\mathbf{A} \nabla)
$$

where $\mathbf{A}(x, t)=\left(a^{i j}(x, t)\right)_{i, j=1}^{n}$ is a real symmetric matrix such that for some $\Lambda \geq \lambda>0$,

$$
\lambda|\xi|^{2} \leq a^{i j}(x, t) \xi_{i} \xi_{j} \leq \Lambda|\xi|^{2}, \quad \forall \xi \in \mathbb{R}^{n} .
$$

Here we work with backward parabolic operators because it is more convenient in this context. A function $u$ satisfies that

$$
|P u| \leq N(|u|+|\nabla u|)
$$

and the growth condition

$$
|u(x, t)| \leq N e^{N|x|^{\alpha}}
$$

${ }^{*}$ Center for Applied Mathematics, Tianjin University, Tianjin 300072, PR China; E-mail: jackwu@amss.ac.cn

${ }^{\dagger}$ Hua Loo-Keng Key Laboratory of Mathematics, Institute of Mathematics, AMSS; School of Mathematical Sciences, UCAS, Beijing 100190, PR China; E-mail: lqzhang@math.ac.cn 
for some $\alpha \in[0,2]$, or a weaker condition

$$
e^{-N|x|^{\alpha}} u(x, t) \in L^{2}\left(\mathbb{R}^{n} \times[0,1]\right) .
$$

The backward uniqueness problem is: suppose

$$
u(x, 0)=0, \quad \forall x \in \mathbb{R}^{n},
$$

does $u$ vanish identically in $\mathbb{R}^{n} \times[0,1]$ ?

Here we set $\alpha \in[0,2]$ because the classical examples of Tychonoff [1] show that the backward uniqueness fails when $\alpha>2$.

The backward uniqueness problem has a natural background in the control theory for PDEs. It also appeared in the regularity theory of parabolic equations, for example, it was applied to prove the full regularity of $L_{3, \infty}$-solutions of the 3-dimensional Navier-Stokes equations [2].

When $P$ is with constant coefficients, i.e., the backward heat operator, there are already many results in various domains, such as the exterior domain [3], the half space [4] and some cones [6, 7, 8].

When $P$ is a general operator with variable coefficients, the results for the LandisOleinik conjecture [9, 11], backward uniqueness in the half space [10] and unique continuation [5] imply that the backward uniqueness in the whole space is valid under the Lipschitz conditions

$$
\left|\nabla_{x} a^{i j}(x, t)\right|+\left|\partial_{t} a^{i j}(x, t)\right| \leq M,
$$

and the decay at infinity conditions

$$
\left|\nabla_{x} a^{i j}(x, t)\right| \leq E|x|^{-1}, \text { where } E<E_{0}(n, \Lambda, \lambda) .
$$

Moreover, both conditions (21) and (3) are almost optimal for the backward uniqueness in the half space when the growth rate of $u$ is quadratic exponential (i.e. $\alpha=2$ ), which could be seen from the counter examples constructed by the authors in [10].

On the other hand, the classical result of Lions and Malgrange [12] showed that the backward uniqueness is valid if $u$ lies in the space

$$
\mathcal{H}:=H^{1}\left((0,1), L^{2}\left(\mathbb{R}_{x}^{n}\right)\right) \cap L^{2}\left((0,1), H^{2}\left(\mathbb{R}_{x}^{n}\right)\right)
$$

and

$$
a^{i j}(x, t) \in \operatorname{Lip}\left([0,1], L^{\infty}\left(\mathbb{R}_{x}^{n}\right)\right) .
$$

In this paper we will prove a result which extends the above two results in some ways. We observe that there is a link between the decay rate of $\left|\nabla_{x} a^{i j}(x, t)\right|$ and the exponential growth rate of $u$. We denote

$$
\langle x\rangle=\sqrt{1+|x|^{2}}, \quad \beta=\max \{0, \alpha-1\} .
$$

Our main result is the following.

Theorem 1.1. Suppose $\left\{a^{i j}\right\}$ satisfy (1), and for some constants $E, M, N>0$,

$$
\left|\nabla_{x} a^{i j}(x, t)\right|+\left|\partial_{t} a^{i j}(x, t)\right| \leq M, \quad\left|\nabla_{x} a^{i j}(x, t)\right| \leq E\langle x\rangle^{-\beta}
$$


in $\mathbb{R}^{n} \times[0,1]$. Assume that u satisfies

$$
|P u| \leq N(|u|+|\nabla u|)
$$

and

$$
|u(x, t)| \leq N e^{N|x|^{\alpha}} \quad \text { or } \quad e^{-N|x|^{\alpha}} u(x, t) \in L^{2}\left(\mathbb{R}^{n} \times[0,1]\right) .
$$

Then if $u(x, 0)=0$ in $\mathbb{R}^{n}$, u vanishes identically in $\mathbb{R}^{n} \times[0,1]$.

\section{Remark 1.2.}

1. When $\alpha \in[0,1]$, Theorem 1.1 tells us that the Lipschitz conditions (2) are sufficient for the backward uniqueness even if $|u(x, t)| \leq N e^{N|x|}$ or $e^{-N|x|} u(x, t) \in L^{2}\left(\mathbb{R}^{n} \times\right.$ $[0,1])$. This extends the result of Lions and Malgrange [12] in some ways.

2. When $\alpha=2$, it required the smallness of $E$ for the backward uniqueness in the half space [10]. However as for the whole space, we don't require such condition.

To prove our result we need the following Carleman inequality.

Proposition 1.3. Suppose $\left\{a^{i j}\right\}$ satisfy (1) and (4). For any $v \in C_{0}^{\infty}\left(\mathbb{R}^{n} \times(0,1)\right)$ and any $\gamma>0$, we have

$$
\begin{array}{r}
\int_{\mathbb{R}^{n} \times(0,1)} e^{2 \gamma\left(t^{-K}-1\right)-\frac{b\langle x\rangle^{\alpha}+K}{t}}\left(|v|^{2}+|\nabla v|^{2}\right) d x d t \\
\leq \int_{\mathbb{R}^{n} \times(0,1)} e^{2 \gamma\left(t^{-K}-1\right)-\frac{b\langle x\rangle^{\alpha}+K}{t}}|P v|^{2} d x d t
\end{array}
$$

where $b=\frac{1}{8 \Lambda}$ and $K=K(n, \Lambda, \lambda, M, E, \alpha)$.

It is worthwhile to mention [13, 14] and related results, which discuss the backward uniqueness problem when $u \in \mathcal{H}$ and $a^{i j}(x, t)$ are non-Lipschitz. However, here we just assume that $u$ satisfies (6).

The paper is organized as follows. First we use Carleman inequality (7) to prove Theorem 1.1, then we prove this Carleman inequality.

\section{Proof of the main result}

In this section, we prove Theorem 1.1. First, we extend $u$ and $a^{i j}$ by the following way:

$$
\begin{aligned}
u(x, t) & =0, & & \text { if } t<0 ; \\
a^{i j}(x, t) & =a^{i j}(x, 0), & & \text { if } t<0 .
\end{aligned}
$$

The next lemma implies Theorem 1.1 immediately.

Lemma 2.1. Suppose $\left\{a^{i j}\right\}$ and $u$ are the same as those in Theorem 1.1. Then there exists $T_{1}=T_{1}(\Lambda, N)>0$, such that $u(x, t) \equiv 0$ in $\mathbb{R}^{n} \times\left(0, T_{1}\right)$. 
Proof. We use Carleman inequality (7) to prove this lemma, mainly following the arguments of the corresponding parts in [3] and [10]. We just give the proof for the case that $|u(x, t)| \leq N e^{N|x|^{\alpha}}$, since the proof of the other case is similar.

Without loss of generality, we assume that $\alpha \in[1,2]$.

Step 1. By the regularity theory for solutions of parabolic equations, we have

$$
|u(x, t)|+|\nabla u(x, t)| \leq C(n, \Lambda, \lambda, M, N) e^{2 N|x|^{\alpha}}
$$

when $(x, t) \in \mathbb{R}^{n} \times\left(0, \frac{1}{2}\right)$. In the following, we always denote $C=C(n, \Lambda, \lambda, M, N)$. Let

$$
\tau=\min \left\{1, \frac{1}{2 N}, \frac{b}{8 N}\right\}
$$

We denote

$$
\tilde{u}(x, t)=u\left(\tau x, \tau^{2}\left(t-\frac{1}{2}\right)\right)
$$

and

$$
\tilde{a}^{i j}(x, t)=a^{i j}\left(\tau x, \tau^{2}\left(t-\frac{1}{2}\right)\right)
$$

for $(x, t) \in \mathbb{R}^{n} \times(0,1)$. Then it is easy to see that

$$
\left|\nabla_{x} \tilde{a}^{i j}(x, t)\right|+\left|\partial_{t} \tilde{a}^{i j}(x, t)\right| \leq \tau M \leq M
$$

and

$$
\left|\nabla_{x} \tilde{a}^{i j}(x, t)\right|=\tau\left|\nabla_{x} a^{i j}\left(\tau x, \tau^{2}\left(t-\frac{1}{2}\right)\right)\right| \leq \tau E\langle\tau x\rangle^{-\beta} \leq E \tau^{1-\beta}\langle x\rangle^{-\beta} \leq E\langle x\rangle^{-\beta} .
$$

We denote

$$
\tilde{P}=\partial_{t}+\partial_{i}\left(\tilde{a}^{i j} \partial_{j}\right)
$$

then by (5) and (9) we have

$$
|\tilde{P} \tilde{u}| \leq \tau N(|\tilde{u}|+|\nabla \tilde{u}|) \leq \frac{1}{2}(|\tilde{u}|+|\nabla \tilde{u}|) .
$$

By (8) and (9) we have

$$
|\tilde{u}(x, t)|+|\nabla \tilde{u}(x, t)| \leq C e^{2 N \tau^{\alpha}|x|^{\alpha}} \leq C e^{2 N \tau|x|^{\alpha}} \leq C e^{\frac{b}{4}\langle x\rangle^{\alpha}}
$$

when $(x, t) \in \mathbb{R}^{n} \times(0,1)$, and

$$
\tilde{u}(x, t)=0
$$

when $(x, t) \in \mathbb{R}^{n} \times\left(0, \frac{1}{2}\right]$.

Step 2. In order to apply Carleman inequality (7), we choose two smooth cut-off functions such that

$$
\eta_{1}(|x|)= \begin{cases}1, & \text { if }|x|<R \\ 0, & \text { if }|x|>R+1\end{cases}
$$

where $R$ is large enough, and

$$
\eta_{2}(t)= \begin{cases}1, & \text { if } t<\frac{3}{4} \\ 0, & \text { if } t>\frac{7}{8}\end{cases}
$$


Let $\eta=\eta_{1} \eta_{2}$ and $v=\eta \tilde{u}$. Then supp $v \subset \mathbb{R}^{n} \times(0,1)$. By (10) we have

$$
\begin{aligned}
|\tilde{P} v| & =\left|\eta \tilde{P} \tilde{u}+\tilde{u} \tilde{P} \eta+2 \tilde{a}^{i j} \partial_{i} \eta \partial_{j} \tilde{u}\right| \\
& \leq \frac{1}{2} \eta(|\tilde{u}|+|\nabla \tilde{u}|)+C(|\tilde{u}|+|\nabla \tilde{u}|)\left(\left|\partial_{t} \eta\right|+|\nabla \eta|+\left|\nabla^{2} \eta\right|\right) \\
& \leq \frac{1}{2}(|v|+|\nabla v|)+C \chi_{\Omega}(|\tilde{u}|+|\nabla \tilde{u}|),
\end{aligned}
$$

where $\chi$ is the characteristic function and

$$
\Omega=\left\{0<\eta<1, \frac{1}{2}<t<1\right\} .
$$

Moreover,

$$
\begin{aligned}
\Omega & =\left\{0<\eta_{1}<1, \eta_{2}>0, \frac{1}{2}<t<1\right\} \cup\left\{\eta_{1}=1,0<\eta_{2}<1, \frac{1}{2}<t<1\right\} \\
& \left.=\left\{R<|x|<R+1, \frac{1}{2}<t<\frac{7}{8}\right)\right\} \cup\left\{|x|<R, \frac{3}{4}<t<\frac{7}{8}\right\} .
\end{aligned}
$$

Step 3. We apply Carleman inequality (7) for $\tilde{P}$ and $v$, then

$$
\begin{aligned}
J & \equiv \int_{\mathbb{R}^{n} \times(0,1)} e^{2 \gamma\left(t^{-K}-1\right)-\frac{b\langle x\rangle^{\alpha}+K}{t}}\left(|v|^{2}+|\nabla v|^{2}\right) d x d t \\
& \leq \int_{\mathbb{R}^{n} \times(0,1)} e^{2 \gamma\left(t^{-K}-1\right)-\frac{b\langle x\rangle^{\alpha}+K}{t}}|\tilde{P} v|^{2} d x d t .
\end{aligned}
$$

By (13) we have

$$
J \leq \frac{3}{4} J+C \int_{\Omega} e^{2 \gamma\left(t^{-K}-1\right)-\frac{b\langle x\rangle^{\alpha}+K}{t}}(|\tilde{u}|+|\nabla \tilde{u}|)^{2} d x d t
$$

thus

$$
J \leq C \int_{\Omega} e^{2 \gamma\left(t^{-K}-1\right)-\frac{b\langle x\rangle^{\alpha}+K}{t}}(|\tilde{u}|+|\nabla \tilde{u}|)^{2} d x d t
$$

By (11) we obtain

$$
\begin{aligned}
J & \leq C \int_{\Omega} e^{2 \gamma\left(t^{-K}-1\right)-\frac{b}{2}\langle x\rangle^{\alpha}} d x d t \\
& =C\left(\int_{\left\{R<|x|<R+1, \frac{1}{2}<t<\frac{7}{8}\right\}}+\int_{\left\{|x|<R, \frac{3}{4}<t<\frac{7}{8}\right\}}\right) e^{2 \gamma\left(t^{-K}-1\right)-\frac{b}{2}\langle x\rangle^{\alpha}} d x d t \\
& \equiv J_{1}+J_{2} .
\end{aligned}
$$

Step 4. Now we estimate both sides of the above inequality.

Estimate of $J_{1}$.

$$
\begin{aligned}
J_{1} & \leq C e^{2 \gamma\left(2^{K}-1\right)} \int_{\{R<|x|<R+1\}} e^{-\frac{b}{2}\langle x\rangle^{\alpha}} d x \\
& \leq C e^{2^{K+1} \gamma-\frac{b}{4} R^{\alpha}} \int_{\{R<|x|<R+1\}} e^{-\frac{b}{4}\langle x\rangle^{\alpha}} d x \\
& \leq C e^{2^{K+1} \gamma-\frac{b}{4} R^{\alpha}} .
\end{aligned}
$$




\section{Estimate of $J_{2}$.}

$$
J_{2} \leq C e^{2 \gamma\left[\left(\frac{3}{4}\right)^{-K}-1\right]} \int_{\{|x|<R\}} e^{-\frac{b}{2}\langle x\rangle^{\alpha}} d x \leq C e^{2 \gamma\left[\left(\frac{3}{4}\right)^{-K}-1\right]} .
$$

Estimate of $J$. For an arbitrary $l \in\left(\frac{1}{2}, \frac{3}{4}\right)$,

$$
\begin{aligned}
J & \geq \int_{\left\{|x|<R, \frac{1}{2}<t<l\right\}} e^{2 \gamma\left(t^{-K}-1\right)-\frac{b\langle x\rangle^{\alpha}+K}{t}}\left(|\tilde{u}|^{2}+|\nabla \tilde{u}|^{2}\right) d x d t \\
& \geq e^{2 \gamma\left(l^{-K}-1\right)} \int_{\left\{|x|<R, \frac{1}{2}<t<l\right\}} e^{-\frac{b\langle x\rangle^{\alpha}+K}{t}}\left(|\tilde{u}|^{2}+|\nabla \tilde{u}|^{2}\right) d x d t .
\end{aligned}
$$

We combine (14)-(17), then we have

$$
\begin{aligned}
& \int_{\left\{|x|<R, \frac{1}{2}<t<l\right\}} e^{-\frac{b\langle x\rangle^{\alpha}+K}{t}}\left(|\tilde{u}|^{2}+|\nabla \tilde{u}|^{2}\right) d x d t \\
\leq & C e^{2 \gamma\left(1-l^{-K}\right)}\left(e^{2^{K+1} \gamma-\frac{b}{4} R^{\alpha}}+e^{2 \gamma\left[\left(\frac{3}{4}\right)^{-K}-1\right]}\right) .
\end{aligned}
$$

In the above inequality, we fix $\gamma$ and let $R \rightarrow \infty$, then we obtain

$$
\int_{\mathbb{R}^{n} \times\left(\frac{1}{2}, l\right)} e^{-\frac{b\langle x\rangle^{\alpha}+K}{t}}\left(|\tilde{u}|^{2}+|\nabla \tilde{u}|^{2}\right) d x d t \leq C e^{2 \gamma\left[\left(\frac{3}{4}\right)^{-K}-l^{-K}\right]} .
$$

Now we fix $l$ and let $\gamma \rightarrow \infty$, then we have $\tilde{u}(x, t) \equiv 0$ in $\mathbb{R}^{n} \times\left(\frac{1}{2}, l\right)$.

Since $l$ is an arbitrary number in $\left(\frac{1}{2}, \frac{3}{4}\right)$, then $\tilde{u}(x, t) \equiv 0$ in $\mathbb{R}^{n} \times\left(\frac{1}{2}, \frac{3}{4}\right)$. That is, $u(x, t) \equiv 0$ in $\mathbb{R}^{n} \times\left(0, \frac{\tau^{2}}{4}\right)$.

Finally we let

$$
T_{1}=\frac{\tau^{2}}{4}=\min \left\{\frac{1}{4}, \frac{1}{16 N^{2}}, \frac{b^{2}}{256 N^{2}}\right\},
$$

then $T_{1}=T_{1}(\Lambda, N)$ and $u(x, t) \equiv 0$ in $\mathbb{R}^{n} \times\left(0, T_{1}\right)$.

Thus we proved this lemma.

\section{Proof of the Carleman inequality}

In this section, we prove Carleman inequality (7). We need two lemmas in our proof. The first one is due to Escauriaza and Fernández [5] (see also [11, Corollary 3.2]). In the following, we denote

$$
\tilde{\Delta}=\partial_{i}\left(a^{i j} \partial_{j}\right)
$$

Lemma 3.1. Suppose $F$ is differentiable, $F_{0}$ and $G$ are twice differentiable and $G>0$. Then the following identity holds for any $v \in C_{0}^{\infty}\left(\mathbb{R}^{n} \times(0, T)\right)$ :

$$
\begin{aligned}
& \frac{1}{2} \int_{\mathbb{R}^{n} \times(0, T)} v^{2} M_{0} G d x d t+\int_{\mathbb{R}^{n} \times(0, T)}\left\langle\left[2 \mathbf{D}_{G}+\left(\frac{\partial_{t} G-\tilde{\Delta} G}{G}-F\right) \mathbf{A}\right] \nabla v, \nabla v\right\rangle G d x d t \\
& -\int_{\mathbb{R}^{n} \times(0, T)} v\left\langle\mathbf{A} \nabla v, \nabla\left(F-F_{0}\right)\right\rangle G d x d t=2 \int_{\mathbb{R}^{n} \times(0, T)} L v(P v-L v) G d x d t,
\end{aligned}
$$


where

$$
\begin{gathered}
L v=\partial_{t} v-\langle\mathbf{A} \nabla v, \nabla \log G\rangle+\frac{F v}{2}, \\
M_{0}=\partial_{t} F+F\left(\frac{\partial_{t} G-\tilde{\Delta} G}{G}-F\right)+\tilde{\Delta} F_{0}-\left\langle\mathbf{A} \nabla\left(F-F_{0}\right), \nabla \log G\right\rangle,
\end{gathered}
$$

and

$$
\mathbf{D}_{G}^{i j}=a^{i k} \partial_{k l}(\log G) a^{l j}+\frac{\partial_{l}(\log G)}{2}\left(a^{k i} \partial_{k} a^{l j}+a^{k j} \partial_{k} a^{l i}-a^{k l} \partial_{k} a^{i j}\right)+\frac{1}{2} \partial_{t} a^{i j}
$$

The second one is concerned with the properties of mollified $\left\{a^{i j}\right\}$.

Lemma 3.2. Suppose $\left\{a^{i j}\right\}$ satisfy (11) and (4). Let

$$
a_{\epsilon}^{i j}(x, t)=\int_{\mathbb{R}^{n}} a^{i j}(x-y, t) \phi_{\epsilon}(y) d y
$$

where $\phi$ is a mollifier and $\epsilon=\frac{1}{2}$. Then $\left\{a_{\epsilon}^{i j}\right\}$ satisfy:

1) $\lambda|\xi|^{2} \leq a_{\epsilon}^{i j}(x, t) \xi_{i} \xi_{j} \leq \Lambda|\xi|^{2}, \quad \forall \xi \in \mathbb{R}^{n}$

2) $\left|\nabla a_{\epsilon}^{i j}(x, t)\right| \leq M ; \quad\left|\nabla a_{\epsilon}^{i j}(x, t)\right| \leq 2 E\langle x\rangle^{-\beta} \quad$ when $|x| \geq 1$;

3) $\left|a_{\epsilon}^{i j}(x, t)-a^{i j}(x, t)\right| \leq 2 \Lambda ; \quad\left|a_{\epsilon}^{i j}(x, t)-a^{i j}(x, t)\right| \leq E\langle x\rangle^{-\beta}$ when $|x| \geq 1$;

4) $\left|\partial_{k l} a_{\epsilon}^{i j}(x, t)\right| \leq c(n) M ; \quad\left|\partial_{k l} a_{\epsilon}^{i j}(x, t)\right| \leq c(n) E\langle x\rangle^{-\beta} \quad$ when $|x| \geq 1$.

\section{Proof of Lemma 3.2.}

1) Obvious.

2)

$$
\left|\nabla a_{\epsilon}^{i j}(x, t)\right| \leq \int_{\mathbb{R}^{n}}\left|\nabla a^{i j}(x-y, t)\right| \phi_{\epsilon}(y) d y \leq M \int_{\mathbb{R}^{n}} \phi_{\epsilon}(y) d y=M,
$$

and when $|x| \geq 1$,

$$
\left|\nabla a_{\epsilon}^{i j}(x, t)\right| \leq \int_{\mathbb{R}^{n}}\left|\nabla a^{i j}(x-y, t)\right| \phi_{\epsilon}(y) d y \leq E \int_{\mathbb{R}^{n}}\langle x-y\rangle^{-\beta} \phi_{\epsilon}(y) d y .
$$

Since $|x| \geq 1$ and $|y| \leq \frac{1}{2}$, then $\langle x-y\rangle \geq \frac{1}{2}\langle x\rangle$ and thus

$$
\left|\nabla a_{\epsilon}^{i j}(x, t)\right| \leq E 2^{\beta}\langle x\rangle^{-\beta} \int_{\mathbb{R}^{n}} \phi_{\epsilon}(y) d y \leq 2 E\langle x\rangle^{-\beta} .
$$

3) The first part is obvious. We only need to prove the second one.

$$
\begin{aligned}
\left|a_{\epsilon}^{i j}(x, t)-a^{i j}(x, t)\right| & \leq \int_{\mathbb{R}^{n}}\left|a^{i j}(x-y, t)-a^{i j}(x, t)\right| \phi_{\epsilon}(y) d y \\
& \leq \int_{\mathbb{R}^{n}}\left|\nabla a^{i j}(x-\theta y, t)\right||y| \phi_{\epsilon}(y) d y, \quad(0<\theta<1)
\end{aligned}
$$

and when $|x| \geq 1$,

$$
\left|a_{\epsilon}^{i j}(x, t)-a^{i j}(x, t)\right| \leq \frac{E}{2} \int_{\mathbb{R}^{n}}\langle x-\theta y\rangle^{-\beta} \phi_{\epsilon}(y) d y \leq E 2^{\beta-1}\langle x\rangle^{-\beta} \int_{\mathbb{R}^{n}} \phi_{\epsilon}(y) d y \leq E\langle x\rangle^{-\beta}
$$


4)

$$
\begin{aligned}
\left|\partial_{k l} a_{\epsilon}^{i j}(x, t)\right| & \leq \int_{\mathbb{R}^{n}}\left|\partial_{k} a^{i j}(x-y, t) \| \partial_{l} \phi_{\epsilon}(y)\right| d y \\
& \leq \epsilon^{-n-1} \int_{\mathbb{R}^{n}}\left|\partial_{k} a^{i j}(x-y, t) \|\left(\partial_{l} \phi\right)\left(\frac{y}{\epsilon}\right)\right| d y \\
& \leq \frac{M}{\epsilon}\left\|\partial_{l} \phi\right\|_{L^{1}} \leq 2 M\|\nabla \phi\|_{L^{1}},
\end{aligned}
$$

and when $|x| \geq 1$,

$$
\begin{aligned}
\left|\partial_{k l} a_{\epsilon}^{i j}(x, t)\right| & \leq \epsilon^{-n-1} \int_{\mathbb{R}^{n}}\left|\partial_{k} a^{i j}(x-y, t) \|\left(\partial_{l} \phi\right)\left(\frac{y}{\epsilon}\right)\right| d y \\
& \leq \epsilon^{-n-1} E \int_{\mathbb{R}^{n}}\langle x-y\rangle^{-\beta}\left|\left(\partial_{l} \phi\right)\left(\frac{y}{\epsilon}\right)\right| d y \\
& \leq \frac{E 2^{\beta}}{\epsilon}\langle x\rangle^{-\beta}\left\|\partial_{l} \phi\right\|_{L^{1}} \leq 4 E\langle x\rangle^{-\beta}\|\nabla \phi\|_{L^{1}} .
\end{aligned}
$$

Now we begin to prove Proposition 1.3 .

Proof of Proposition 1.3. In (18), we let

$$
G=e^{2 \gamma\left(t^{-K}-1\right)-\frac{b\langle x\rangle^{\alpha}+K}{t}},
$$

then

$$
\frac{\partial_{t} G-\tilde{\Delta} G}{G}=\frac{b\langle x\rangle^{\alpha}-\alpha^{2} b^{2}\langle x\rangle^{2 \alpha-4} a^{i j} x_{i} x_{j}+K}{t^{2}}+\frac{\alpha b\langle x\rangle^{\alpha-2}\left(a^{i i}+\partial_{k} a^{k l} x_{l}\right)}{t}-2 \gamma K t^{-K-1} .
$$

Let

$$
F=\frac{b\langle x\rangle^{\alpha}-\alpha^{2} b^{2}\langle x\rangle^{2 \alpha-4} a^{i j} x_{i} x_{j}+K}{t^{2}}+\frac{\alpha b\langle x\rangle^{\alpha-2} a^{i i}-d}{t}-2 \gamma K t^{-K-1},
$$

where $d$ is a positive constant to be determined, and

$$
F_{0}=\frac{b\langle x\rangle^{\alpha}-\alpha^{2} b^{2}\langle x\rangle^{2 \alpha-4} a_{\epsilon}^{i j} x_{i} x_{j}+K}{t^{2}}+\frac{\alpha b\langle x\rangle^{\alpha-2} a_{\epsilon}^{i i}-d}{t}-2 \gamma K t^{-K-1} .
$$

We denote by $\mathbf{I}_{n}$ the identity matrix of $\mathbb{R}^{n}, C$ are generic constants depending on $n, \Lambda, \lambda, M, E$ and $\alpha$ in the following arguments. We need some estimates which we list in the following lemma.

Lemma 3.3. Set $b=\frac{1}{8 \Lambda}$ and $d=\frac{K}{4}$. For $K \geq K_{0}(n, \Lambda, \lambda, M, E, \alpha)$, we have

$$
\begin{aligned}
2 \mathbf{D}_{G}+\left(\frac{\partial_{t} G-\tilde{\Delta} G}{G}-F\right) \mathbf{A} & \geq \frac{\lambda K}{8 t} \mathbf{I}_{n} ; \\
\partial_{t} F+F\left(\frac{\partial_{t} G-\tilde{\Delta} G}{G}-F\right) & \geq \frac{b K\langle x\rangle^{\alpha}}{16 t^{3}} ; \\
\left|\tilde{\triangle} F_{0}\right| & \leq \frac{C\langle x\rangle^{\alpha}}{t^{2}} ; \\
\left|\nabla\left(F-F_{0}\right)\right| & \leq \frac{C\langle x\rangle^{\alpha-1}}{t^{2}} .
\end{aligned}
$$


We will prove this lemma later.

First by (20) we have

$$
\begin{aligned}
& \int_{\mathbb{R}^{n} \times(0,1)}\left\langle\left[2 \mathbf{D}_{G}+\left(\frac{\partial_{t} G-\tilde{\Delta} G}{G}-F\right) \mathbf{A}\right] \nabla v, \nabla v\right\rangle G d x d t \\
\geq & \frac{\lambda K}{8} \int_{\mathbb{R}^{n} \times(0,1)} \frac{|\nabla v|^{2}}{t} G d x d t .
\end{aligned}
$$

Next we estimate $M_{0}$. By (23) and

$$
\nabla \log G=-\frac{\alpha b}{t}\langle x\rangle^{\alpha-2} x
$$

we have

$$
\left|\left\langle\mathbf{A} \nabla\left(F-F_{0}\right), \nabla \log G\right\rangle\right| \leq \Lambda\left|\nabla\left(F-F_{0}\right)\right||\nabla \log G| \leq \frac{C\langle x\rangle^{2 \alpha-2}}{t^{3}} \leq \frac{C\langle x\rangle^{\alpha}}{t^{3}} .
$$

Then by (21), (22) and (25) we have

$$
\begin{aligned}
M_{0} & =\partial_{t} F+F\left(\frac{\partial_{t} G-\tilde{\Delta} G}{G}-F\right)+\tilde{\Delta} F_{0}-\left\langle A \nabla\left(F-F_{0}\right), \nabla \log G\right\rangle \\
& \geq\left(\frac{b K}{16}-C\right) \frac{\langle x\rangle^{\alpha}}{t^{3}},
\end{aligned}
$$

thus

$$
\frac{1}{2} \int_{\mathbb{R}^{n} \times(0,1)} v^{2} M_{0} G d x d t \geq\left(\frac{b K}{32}-C\right) \int_{\mathbb{R}^{n} \times(0,1)} \frac{\langle x\rangle^{\alpha}}{t^{3}} v^{2} G d x d t .
$$

By the Cauchy inequality and (23) we have

$$
\begin{aligned}
& \left|\int_{\mathbb{R}^{n} \times(0,1)} v\left\langle\mathbf{A} \nabla v, \nabla\left(F-F_{0}\right)\right\rangle G d x d t\right| \\
\leq \Lambda & \int_{\mathbb{R}^{n} \times(0,1)}\left|\nabla\left(F-F_{0}\right)\right||v||\nabla v| G d x d t \\
\leq & C \int_{\mathbb{R}^{n} \times(0,1)} \frac{\langle x\rangle^{\alpha-1}}{t^{2}}|v||\nabla v| G d x d t \\
\leq & C \int_{\mathbb{R}^{n} \times(0,1)} \frac{\langle x\rangle^{2 \alpha-2}}{t^{3}} v^{2} G d x d t+C \int_{\mathbb{R}^{n} \times(0,1)} \frac{|\nabla v|^{2}}{t} G d x d t \\
\leq & \int_{\mathbb{R}^{n} \times(0,1)} \frac{\langle x\rangle^{\alpha}}{t^{3}} v^{2} G d x d t+C \int_{\mathbb{R}^{n} \times(0,1)} \frac{|\nabla v|^{2}}{t} G d x d t .
\end{aligned}
$$

Finally, by (18), (24), (26), (27) and the Cauchy inequality, we have

$$
\int_{\mathbb{R}^{n} \times(0,1)}|P u|^{2} G d x d t \geq\left(\frac{b K}{32}-C\right) \int_{\mathbb{R}^{n} \times(0,1)} \frac{\langle x\rangle^{\alpha}}{t^{3}} v^{2} G d x d t+\left(\frac{\lambda K}{8}-C\right) \int_{\mathbb{R}^{n} \times(0,1)} \frac{|\nabla v|^{2}}{t} G d x d t,
$$

if we choose $K \geq K_{0}(n, \Lambda, \lambda, M, E, \alpha)$ large enough, we obtain

$$
\int_{\mathbb{R}^{n} \times(0,1)}|P v|^{2} G d x d t \geq \int_{\mathbb{R}^{n} \times(0,1)}\left(v^{2}+|\nabla v|^{2}\right) G d x d t
$$

Thus we proved Carleman inequality (17). 
There is only Lemma 3.3 left to be proven.

Proof of Lemma 3.3. We estimate them one by one.

Estimate of $2 \mathbf{D}_{G}+\left(\frac{\partial_{t} G-\tilde{\Delta} G}{G}-F\right) \mathbf{A}$.

By direct calculations we have

$$
\begin{aligned}
& 2 \mathbf{D}_{G}+\left(\frac{\partial_{t} G-\tilde{\Delta} G}{G}-F\right) \mathbf{A} \\
= & -\frac{2 \alpha b}{t}\langle x\rangle^{\alpha-2} \mathbf{A}^{2}+\frac{2 \alpha(2-\alpha) b}{t}\langle x\rangle^{\alpha-4} \mathbf{A} x(\mathbf{A} x)^{\prime} \\
& -\frac{\alpha b}{t}\langle x\rangle^{\alpha-2} x_{l}\left(a^{k i} \partial_{k} a^{l j}+a^{k j} \partial_{k} a^{l i}-a^{k l} \partial_{k} a^{i j}-a^{i j} \partial_{k} a^{k l}\right)+\partial_{t} a^{i j}+\frac{d}{t} \mathbf{A} \\
\geq & -\frac{2 \alpha b \Lambda^{2}}{t}\langle x\rangle^{\alpha-2} \mathbf{I}_{n}-\frac{\alpha b}{t}\langle x\rangle^{\alpha-2} x_{l}\left(a^{k i} \partial_{k} a^{l j}+a^{k j} \partial_{k} a^{l i}-a^{k l} \partial_{k} a^{i j}-a^{i j} \partial_{k} a^{k l}\right)+\partial_{t} a^{i j}+\frac{\lambda d}{t} \mathbf{I}_{n} .
\end{aligned}
$$

Next we estimate the lower bounds of the matrices in the right side of the above inequality. We just need to estimate matrix $x_{l} a^{k i} \partial_{k} a^{l j}$ and $\partial_{t} a^{i j}$. For any $\xi \in \mathbb{R}^{n}$,

$$
\left|x_{l} a^{k i} \partial_{k} a^{l j} \xi_{i} \xi_{j}\right| \leq n^{2} \Lambda E|x|\langle x\rangle^{-\beta} \sum_{i, j}\left|\xi_{i}\right|\left|\xi_{j}\right| \leq n^{3} \Lambda E\langle x\rangle^{1-\beta}|\xi|^{2}
$$

then

$$
-n^{3} \Lambda E\langle x\rangle^{1-\beta} \mathbf{I}_{n} \leq x_{l} a^{k i} \partial_{k} a^{l j} \leq n^{3} \Lambda E\langle x\rangle^{1-\beta} \mathbf{I}_{n}
$$

Similarly,

$$
\left|\partial_{t} a^{i j} \xi_{i} \xi_{j}\right| \leq M \sum_{i, j}\left|\xi_{i}\right|\left|\xi_{j}\right| \leq M n|\xi|^{2}
$$

then

$$
-M n \mathbf{I}_{n} \leq \partial_{t} a^{i j} \leq M n \mathbf{I}_{n}
$$

Thus we have

$$
2 \mathbf{D}_{G}+\left(\frac{\partial_{t} G-\tilde{\Delta} G}{G}-F\right) \mathbf{A} \geq\left(-\frac{2 \alpha b \Lambda^{2}}{t}\langle x\rangle^{\alpha-2}-\frac{4 \alpha b n^{3} \Lambda E}{t}\langle x\rangle^{\alpha-\beta-1}-M n+\frac{\lambda d}{t}\right) \mathbf{I}_{n} .
$$

Notice that $\alpha-2 \leq 0$ and $\alpha-\beta-1 \leq 0$, and if we choose $d=d(n, \Lambda, \lambda, M, E$, $\alpha)$ large enough, then

$$
2 \mathbf{D}_{G}+\left(\frac{\partial_{t} G-\tilde{\Delta} G}{G}-F\right) \mathbf{A} \geq \frac{\lambda d}{2 t} \mathbf{I}_{n} .
$$

Estimate of $\partial_{t} F+F\left(\frac{\partial_{t} G-\tilde{\Delta} G}{G}-F\right)$.

By direct calculations we have

$$
\begin{aligned}
& \partial_{t} F+F\left(\frac{\partial_{t} G-\tilde{\Delta} G}{G}-F\right) \\
= & \frac{\left(d+\alpha b\langle x\rangle^{\alpha-2} \partial_{i} a^{i j} x_{j}-2\right)\left(b\langle x\rangle^{\alpha}-\alpha^{2} b^{2}\langle x\rangle^{2 \alpha-4} a^{i j} x_{i} x_{j}+K\right)}{t^{3}} \\
& -\frac{\alpha^{2} b^{2}\langle x\rangle^{2 \alpha-4} \partial_{t} a^{i j} x_{i} x_{j}+\left(d-\alpha b\langle x\rangle^{\alpha-2} a^{i i}\right)\left(d+\alpha b\langle x\rangle^{\alpha-2} \partial_{i} a^{i j} x_{j}-1\right)}{t^{2}} \\
& +\frac{\alpha b\langle x\rangle^{\alpha-2} \partial_{t} a^{i i}}{t}+2 \gamma K t^{-K-2}\left[K+1-\left(d+\alpha b\langle x\rangle^{\alpha-2} \partial_{i} a^{i j} x_{j}\right)\right] .
\end{aligned}
$$


Notice that

$$
\begin{aligned}
\langle x\rangle^{\alpha-2}\left|\partial_{i} a^{i j} x_{j}\right| & \leq C\langle x\rangle^{\alpha-\beta-2}|x| \leq C\langle x\rangle^{\alpha-\beta-1} \leq C, \\
\langle x\rangle^{2 \alpha-4} a^{i j} x_{i} x_{j} & \leq \Lambda\langle x\rangle^{2 \alpha-4}|x|^{2} \leq \Lambda\langle x\rangle^{2 \alpha-2} \leq \Lambda\langle x\rangle^{\alpha}, \\
\langle x\rangle^{2 \alpha-4}\left|\partial_{t} a^{i j} x_{i} x_{j}\right| & \leq C\langle x\rangle^{2 \alpha-4}|x|^{2} \leq C\langle x\rangle^{\alpha}
\end{aligned}
$$

then we have

$$
\begin{aligned}
& \partial_{t} F+F\left(\frac{\partial_{t} G-\tilde{\Delta} G}{G}-F\right) \\
\geq & \frac{(d-C)\left[\left(b-\alpha^{2} b^{2} \Lambda\right)\langle x\rangle^{\alpha}+K\right]}{t^{3}}-\frac{C\langle x\rangle^{\alpha}+(d+C)^{2}}{t^{2}} \\
& -\frac{C}{t}+2 \gamma K t^{-K-2}(K-d-C) .
\end{aligned}
$$

Recall that $b=\frac{1}{8 \Lambda}$, and thus $\alpha^{2} b^{2} \Lambda \leq 4 b^{2} \Lambda \leq \frac{b}{2}$. If we choose $d$ large enough, then

$$
\begin{aligned}
& \partial_{t} F+F\left(\frac{\partial_{t} G-\tilde{\Delta} G}{G}-F\right) \\
\geq & \frac{(d-C)\left[\frac{b}{2}\langle x\rangle^{\alpha}+K\right]-C\langle x\rangle^{\alpha}-(d+C)^{2}-C}{t^{3}}+2 \gamma K t^{-K-2}(K-2 d) \\
\geq & \frac{\left(\frac{b d}{2}-C\right)\langle x\rangle^{\alpha}+(d-C) K-2 d^{2}}{t^{3}}+2 \gamma K t^{-K-2}(K-2 d) .
\end{aligned}
$$

We choose $d=\frac{K}{4}$, then

$$
\partial_{t} F+F\left(\frac{\partial_{t} G-\tilde{\Delta} G}{G}-F\right) \geq\left(\frac{b K}{8}-C\right) \frac{\langle x\rangle^{\alpha}}{t^{3}}+\gamma K^{2} t^{-K-2} \geq \frac{b K\langle x\rangle^{\alpha}}{16 t^{3}} .
$$

Estimate of $\tilde{\triangle} F_{0}$.

Direct calculations show that

$$
\tilde{\triangle} F_{0}=\frac{b}{t^{2}} \tilde{\triangle}\left(\langle x\rangle^{\alpha}\right)-\frac{\alpha^{2} b^{2}}{t^{2}} \tilde{\triangle}\left(\langle x\rangle^{2 \alpha-4} a_{\epsilon}^{i j} x_{i} x_{j}\right)+\frac{\alpha b}{t} \tilde{\triangle}\left(\langle x\rangle^{\alpha-2} a_{\epsilon}^{i i}\right),
$$

and

$$
\begin{gathered}
\tilde{\triangle}\left(\langle x\rangle^{\alpha}\right)=\alpha\langle x\rangle^{\alpha-2}\left(a^{i i}+\partial_{i} a^{i j} x_{j}\right)+\alpha(\alpha-2)\langle x\rangle^{\alpha-4} a^{i j} x_{i} x_{j}, \\
\tilde{\triangle}\left(\langle x\rangle^{2 \alpha-4} a_{\epsilon}^{i j} x_{i} x_{j}\right)=(2 \alpha-4)(2 \alpha-6)\langle x\rangle^{2 \alpha-8} a^{k l} a_{\epsilon}^{i j} x_{i} x_{j} x_{k} x_{l} \\
+(2 \alpha-4)\langle x\rangle^{2 \alpha-6}\left[\left(\partial_{l} a^{k l} a_{\epsilon}^{i j}+2 a^{k l} \partial_{l} a_{\epsilon}^{i j}\right) x_{i} x_{j} x_{k}+\left(4 a^{k i} a_{\epsilon}^{k j}+a^{k k} a_{\epsilon}^{i j}\right) x_{i} x_{j}\right] \\
+\langle x\rangle^{2 \alpha-4}\left[\left(a^{k l} \partial_{k l} a_{\epsilon}^{i j}+\partial_{k} a^{k l} \partial_{l} a_{\epsilon}^{i j}\right) x_{i} x_{j}+\left(2 \partial_{k} a^{k j} a_{\epsilon}^{i j}+4 a^{k j} \partial_{k} a_{\epsilon}^{i j}\right) x_{i}+2 a^{i j} a_{\epsilon}^{i j}\right], \\
\tilde{\triangle}\left(\langle x\rangle^{\alpha-2} a_{\epsilon}^{i i}\right)= \\
(\alpha-2)(\alpha-4)\langle x\rangle^{\alpha-6} a^{i j} a_{\epsilon}^{k k} x_{i} x_{j} \\
+(\alpha-2)\langle x\rangle^{\alpha-4}\left[\left(\partial_{j} a^{i j} a_{\epsilon}^{k k}+2 a^{i j} \partial_{j} a_{\epsilon}^{k k}\right) x_{i}+a^{i i} a_{\epsilon}^{k k}\right] \\
+\langle x\rangle^{\alpha-2}\left(a^{i j} \partial_{i j} a_{\epsilon}^{k k}+\partial_{i} a^{i j} \partial_{j} a_{\epsilon}^{k k}\right) .
\end{gathered}
$$

By Lemma 3.2 we know that $a^{i j}, \nabla a^{i j}, a_{\epsilon}^{i j}, \nabla a_{\epsilon}^{i j}$ and $\nabla^{2} a_{\epsilon}^{i j}$ are all bounded, then it is easy to verify that

$$
\begin{aligned}
\left|\tilde{\triangle}\left(\langle x\rangle^{\alpha}\right)\right| & \leq C\left(\langle x\rangle^{\alpha-1}+\langle x\rangle^{\alpha-2}\right) \leq C\langle x\rangle^{\alpha-1} ; \\
\left|\tilde{\triangle}\left(\langle x\rangle^{\alpha-4} a_{\epsilon}^{i j} x_{i} x_{j}\right)\right| & \leq C\left(\langle x\rangle^{2 \alpha-4}+\langle x\rangle^{2 \alpha-3}+\langle x\rangle^{2 \alpha-2}\right) \leq C\langle x\rangle^{2 \alpha-2} ; \\
\left|\tilde{\triangle}\left(\langle x\rangle^{\alpha-2} a_{\epsilon}^{i i}\right)\right| & \leq C\left(\langle x\rangle^{\alpha-4}+\langle x\rangle^{\alpha-3}+\langle x\rangle^{\alpha-2}\right) \leq C\langle x\rangle^{\alpha-2} .
\end{aligned}
$$


Finally by (28) and (29) we have

$$
\left|\tilde{\triangle} F_{0}\right| \leq \frac{C}{t^{2}}\left(\langle x\rangle^{\alpha-1}+\langle x\rangle^{2 \alpha-2}+\langle x\rangle^{\alpha-2}\right) \leq \frac{C\langle x\rangle^{\alpha}}{t^{2}} .
$$

Estimate of $\left|\nabla\left(F-F_{0}\right)\right|$.

Since

$$
F-F_{0}=\frac{\alpha^{2} b^{2}}{t^{2}}\langle x\rangle^{2 \alpha-4}\left(a_{\epsilon}^{i j}-a^{i j}\right) x_{i} x_{j}-\frac{\alpha b}{t}\langle x\rangle^{\alpha-2}\left(a_{\epsilon}^{i i}-a^{i i}\right),
$$

then

$$
\begin{aligned}
\nabla\left(F-F_{0}\right)= & \frac{\alpha^{2} b^{2}}{t^{2}}\left[(2 \alpha-4)\langle x\rangle^{2 \alpha-6}\left(a_{\epsilon}^{i j}-a^{i j}\right) x_{i} x_{j} x+2\langle x\rangle^{2 \alpha-4}\left(a_{\epsilon}^{i j}-a^{i j}\right) x_{i} \nabla x_{j}\right. \\
& \left.+\langle x\rangle^{2 \alpha-4}\left(\nabla a_{\epsilon}^{i j}-\nabla a^{i j}\right) x_{i} x_{j}\right] \\
& -\frac{\alpha b}{t}\left[(\alpha-2)\langle x\rangle^{\alpha-4}\left(a_{\epsilon}^{i i}-a^{i i}\right) x+\langle x\rangle^{\alpha-2}\left(\nabla a_{\epsilon}^{i i}-\nabla a^{i i}\right)\right] .
\end{aligned}
$$

Notice that $a^{i j}, \nabla a^{i j}, a_{\epsilon}^{i j}$ and $\nabla a_{\epsilon}^{i j}$ are all bounded, then

$$
\left|\nabla\left(F-F_{0}\right)\right| \leq \frac{C}{t^{2}}\left(\langle x\rangle^{2 \alpha-3}+\langle x\rangle^{2 \alpha-4}\left|\nabla a_{\epsilon}^{i j}-\nabla a^{i j}\right||x|^{2}\right)+\frac{C}{t}\left(\langle x\rangle^{\alpha-3}+\langle x\rangle^{\alpha-2}\right) .
$$

By 2) of (19), when $|x|<1$,

$$
\left|\nabla a_{\epsilon}^{i j}-\nabla a^{i j}\right||x|^{2} \leq 2 M|x|^{2} \leq 2 M,
$$

and when $|x| \geq 1$,

$$
\left|\nabla a_{\epsilon}^{i j}-\nabla a^{i j}\right||x|^{2} \leq\left(2 E\langle x\rangle^{-\beta}+E\langle x\rangle^{-\beta}\right)|x|^{2} \leq 3 E\langle x\rangle^{2-\beta} .
$$

In both cases we have

$$
\left|\nabla a_{\epsilon}^{i j}-\nabla a^{i j}\right||x|^{2} \leq C\langle x\rangle^{2-\beta}
$$

By (30) and (31) we have

$$
\left|\nabla\left(F-F_{0}\right)\right| \leq \frac{C}{t^{2}}\left(\langle x\rangle^{2 \alpha-3}+\langle x\rangle^{2 \alpha-\beta-2}\right)+\frac{C\langle x\rangle^{\alpha-2}}{t}
$$

Since $2 \alpha-\beta-2 \leq \alpha-1$, then

$$
\left|\nabla\left(F-F_{0}\right)\right| \leq \frac{C}{t^{2}}\left(\langle x\rangle^{2 \alpha-3}+\langle x\rangle^{\alpha-1}\right)+\frac{C\langle x\rangle^{\alpha-2}}{t} \leq \frac{C\langle x\rangle^{\alpha-1}}{t^{2}} .
$$

Thus we proved Lemma 3.3 .

Acknowledgments. J. Wu is supported by NSFC under grant 11601373. L. Zhang is partially supported by NSFC under grant 11471320 and 11631008 . 


\section{References}

[1] A. Tychonoff: Théorèmes d'unicité pour l'équation de la chaleur, Mat. Sb. 42 (1935), 199-216.

[2] L. Escauriaza, G. A. Seregin, V. Šverák, $L_{3, \infty}$ solutions to the Navier-Stokes equations and backward uniqueness, Russ. Math. Surv., 58 (2003), no. 2, 211250.

[3] L. Escauriaza, G. A. Seregin, V. Šverák, Backward uniqueness for parabolic equations, Arch. Ration. Mech. Anal., 169 (2003), no. 2, 147-157.

[4] L. Escauriaza, G. A. Seregin, V. Šverák, Backward uniqueness for the heat operator in half-space, Algebra i Analiz 15 (2003), no. 1, 201-214; translation in St. Petersburg Math. J. 15 (2004), no. 1, 139-148.

[5] L. Escauriaza, F. J. Fernández, Unique continuation for parabolic operators, Ark. Mat., 41(2003), no. 1, 35-60.

[6] Lu Li, V. Šverák, Backward uniqueness for the heat equation in cones, Comm. Partial Differential Equations, 37 (2012), no. 8, 1414-1429.

[7] J. Wu, W. Wang, On backward uniqueness for the heat operator in cones, J. Differential Equations, 258 (2015), 224-241.

[8] Rüland, Angkana, On the backward uniqueness property for the heat equation in two-dimensional conical domains, Manuscripta Math. 147 (2015), no. 3-4, 415-436.

[9] Tu A. Nguyen, On a question of Landis and Oleinik, Tran. Amer. Math. Soc., 362 (2010), no. 6, 2875-2899.

[10] J. Wu, L. Zhang, Backward uniqueness for parabolic operators with variable coefficients in a half space, Commun. Contemp. Math., 18 (2016), no. 1, 1550011, $38 \mathrm{pp}$.

[11] J. Wu, L. Zhang, The Landis-Oleinik conjecture in the exterior domain, Adv. Math., 302 (2016), 190-230.

[12] J.-L. Lions, B. Malgrange, Sur l'unicité rétrograde dans les problèmes mixtes paraboliques, Math. Scand. 8 (1960), 277-286.

[13] D. Del Santo, M. Prizzi, Backward uniqueness for parabolic operators whose coefficients are non-Lipschitz continuous in time, J. Math. Pures Appl. 84 (2005), 471-491.

[14] D. Del Santo, C. JÄh, M. Paicu, Backward uniqueness for parabolic operators with non-Lipschitz coefficients, Osaka J. Math. 52 (2015), no. 3, 793-815. 